\title{
Full transmission and reflection of waves propagating through a maze of disorder
}

\author{
Benoît Gérardin, Jérôme Laurent, Arnaud Derode, Claire Prada, and Alexandre Aubry* \\ ESPCI ParisTech, PSL Research University, CNRS, Univ Paris Diderot, \\ Sorbonne Paris Cité, Institut Langevin, UMR 7587, 1 rue Jussieu, F-75005 Paris, France
}

(Dated: October 2, 2018)

\begin{abstract}
Thirty years ago, theorists showed that a properly designed combination of incident waves could be fully transmitted through (or reflected by) a disordered medium, based on the existence of propagation channels which are essentially either closed or open (bimodal law). In this Letter, we study elastic waves in a disordered waveguide and present a direct experimental evidence of the bimodal law. Full transmission and reflection are achieved. The wave-field is monitored by laser interferometry and highlights the interference effects that take place within the scattering medium.
\end{abstract}

PACS numbers: 42.25.Dd, 43.20.Gp, 05.40.-a, 42.25.Bs

Light travelling through thick clouds, electrons conducting through metals or seismic waves in the earth crust are all examples of waves propagating through disordered materials. Energy transport by waves undergoing strong scattering is usually well described by diffusion theory. However, this classical picture neglects interference effects that may resist the influence of disorder. Interference is responsible for fascinating phenomena in mesoscopic physics. On the one hand, it can slow down and eventually stop the diffusion process, giving rise to Anderson localization [1,2]. On the other hand, it can also help waves to find a way through a maze of disorder [3]. Actually, a properly designed combination of incident waves can be completely transmitted through a strongly scattering medium, as suggested by Dorokhov and others more than twenty years ago [4-7]. This prediction has recently received a great deal of attention mostly due to the emergence of wave-front shaping techniques in optics [8].

In order to address the open channels (i.e., to achieve full energy transmission) across a disordered wave guide, one has to perform a complete measurement of the scattering matrix $\mathbf{S}$. The S-matrix relates the input and output of the medium [7]. It fully describes wave propagation across a scattering medium. It can be generally divided into blocks containing transmission and reflection matrices, $\mathbf{t}$ and $\mathbf{r}$, with a certain number $N$ of input and output channels. Initially, random matrix theory (RMT) has been successfully applied to the transport of electrons through chaotic systems and disordered wires [7]. However, the confrontation between theory and experiment has remained quite restrictive since specific input electron states cannot be addressed in practice. On the contrary, a coherent control of the incident wave-field is possible in classical wave physics. Several works have demontrated the ability of measuring the $\mathbf{S}$-matrix, or at least some of its subspaces, in disordered media, whether it be in acoutics [9-11], electromagnetism [12, 13] or optics [14-17].

The existence of open channels has been revealed by investigating the eigenvalues $T$ of the Hermitian matrix $\mathbf{t t}^{\dagger}$. Theoretically, their distribution should follow a bimodal law $[4,5$, 7], exhibiting two peaks. The highest one, around $T \sim 0$, correspond to closed (i.e. strongly reflected) eigenchannels. At the other end of the spectrum $(T \sim 1)$, there are $g$ open eigen- channels. $g=N l^{*} / L$ is the dimensionless conductance [18], $L$ is the sample thickness, and $l^{*}$ is the transport mean free path. By exciting selectively open or closed channels, a nearly complete transmission [19, 20], reflection or absorption [21] of waves can be achieved. It means that a designed wave-front can be fully transmitted or, on the contrary, fully reflected by a scattering medium, which is in total contradiction with the classical diffusion picture. Although some indirect evidence of bimodality have been pointed out experimentally as e.g., the reduction of the shot noise power in electrical conductors [22, 23], these remarkable interference effects have never been directly observed so far. Indeed, the bimodal distribution relies on the conservation of energy (i.e. $\mathbf{S}$ is a unitary matrix). In other words, all the channels should be addressed at the input and measured at the output [24]. In optical experiments, the finite numerical aperture of the illumination and detection systems limits the angular coverage of the input and output channels [25]. In acoustics or electromagnetism, the spatial sampling of measurements has not been sufficient to have access to the full $\mathbf{S}$-matrix so far $[9,10,12]$.

In this Letter, we present experimental measurements of the full S-matrix across a disordered elastic wave guide. To that aim, laser-ultrasonic techniques have been used in order to obtain a satisfying spatial sampling of the field at the input and output of the scattering medium. The unitarity of the $\mathbf{S}-$ matrix is investigated and the eigenvalues of the transmission matrix are shown to follow the expected bimodal distribution. Moreover, full experimental transmission and reflection of waves propagating through disorder are achieved. The wave-fields associated to the open and closed channels are monitored within the scattering medium by laser interferometry to highlight the interference effects operating in each case.

We study here the propagation of elastic waves across a duralumin plate (aluminium alloy) of dimension $500 \times 40 \times 0.5$ $\mathrm{mm}^{3}$ (see Fig. 1). The homogeneous plate is a waveguide in which randomness is introduced by drilling circular holes with diameter $1.5 \mathrm{~mm}$ and concentration $11 \mathrm{~cm}^{-2}$, distributed over a thickness $L=20 \mathrm{~mm}$ and the whole width of the plate $W=40 \mathrm{~mm}$. The $\mathbf{S}$-matrix associated to the disordered slab is measured with the laser-ultrasonic set-up described in 
Fig. 1. Elastic waves are generated in the thermoelastic regime [26] by a pumped diode Nd:YAG laser providing pulses having a $20 \mathrm{~ns}$ duration and $2.5 \mathrm{~mJ}$ of energy. The out-of-plane component of the local vibration of the plate is measured with a heterodyne interferometer [26]. In the frequency range of interest ( 0.32 to $0.37 \mathrm{MHz}, \Delta f=0.05 \mathrm{MHz}$ ), the plate thickness $(d=0.5 \mathrm{~mm})$ is small compared to the wavelength $(\lambda \sim 3.5 \mathrm{~mm})$.

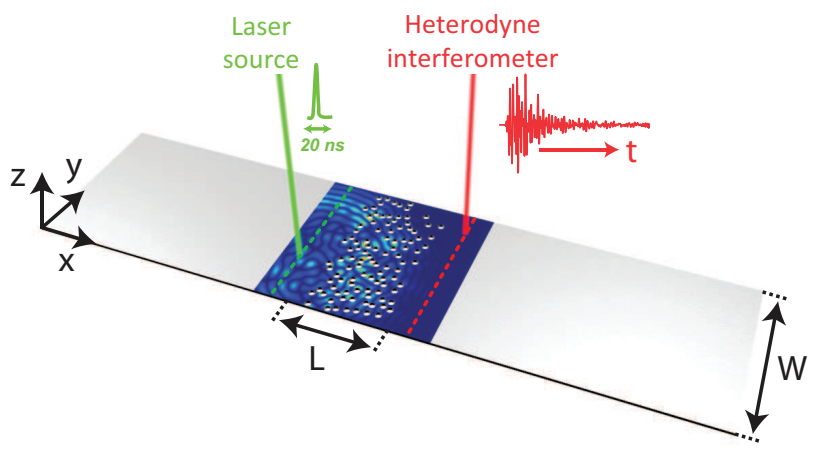

FIG. 1: Experimental set up. The $\mathbf{S}-$ matrix is measured in the timedomain, between two arrays of points placed $5 \mathrm{~mm}$ away from each side of the disordered slab. The array pitch is $0.8 \mathrm{~mm}(\sim \lambda / 4)$. Flexural waves are generated on each point by a pulsed laser via thermo-elastic conversion over a focal spot of $1 \mathrm{~mm}^{2}$. The normal component of the plate vibration is measured with an interferometric optical probe. The laser source and the probe are both mounted on 2D translation stages.

Three types of elastic waves can propagate through the plate: shear horizontal (SH), extensional and flexural waves [27]. For flexural waves, polarization is perpendicular to the plane of the plate whereas it is in-plane for SH and extensional waves. As a consequence, only flexural waves are measured by the heterodyne interferometer. Moreover, as scatterers consist in through holes, there cannot be conversion of $\mathrm{SH} /$ extensional modes into flexural modes via scattering for symmetry reasons [28]. Hence, the experimental setup shown in Fig. 1 allows a measurement of the S-matrix associated to the flexural modes in the plate.

The first step of the experiment consists in measuring the impulse responses between two arrays of points placed on the left and right sides of the disordered slab. Each impulse response is averaged over 128 laser shots in order to reduce additive electronic noise [29]. The array pitch is $0.8 \mathrm{~mm}$ (i.e. $<\lambda / 2$ ) which guarantees a satisfying spatial sampling of the wave field. The impulse responses between any two points of the same array form the time-dependent reflection matrices, $\mathbf{r}$ and $\mathbf{r}^{\prime}$, from left to left and right to right, respectively. The set of impulse responses between the two arrays yield the timedependent transmission matrices $\mathbf{t}$ (from left to right) and $\mathbf{t}^{\prime}$ (from right to left). From these four matrices, one can build the $\mathbf{S}$-matrix in a point-to-point basis,

$$
\mathbf{S}=\left(\begin{array}{ll}
\mathbf{r} & \mathbf{t}^{\prime} \\
\mathbf{t} & \mathbf{r}^{\prime}
\end{array}\right)
$$

A temporal Fourier transform of $\mathbf{S}$ is then performed over a time range $\Delta t=120 \mu$ s that excludes the echoes due to reflections on the ends of the plate. Frequency components that are spaced by more than the correlation frequency $\delta f$ give rise to uncorrelated speckle patterns. In our disordered sample, we have measured $\delta f \sim 0.017 \mathrm{MHz}$. Hence, the number $N_{f}=\Delta f / \delta f$ of independent scattering matrices $\mathbf{S}$ that are obtained over the frequency bandwidth is 3 . The correlation frequency also yields an estimation for the Thouless time $\left(\tau_{D} \sim 1 / \delta f \sim 60 \mu \mathrm{s}\right)$ i.e., the mean time it takes for a wave to cross the sample through its zigzag motion. We check that $\tau_{D} \sim \Delta t / 2$ and that most of the energy has escaped from the sample when the measurement is stopped. The next step of the experimental procedure consists in decomposing the $\mathbf{S}$-matrices in the basis of the flexural modes of the homogeneous plate. These eigenmodes and their wavenumbers have been determined theoretically using the thin elastic plate theory [29-31]. They are normalized so that each of them carries unit energy flux across the plate section. The transformation of the $\mathbf{S}$-matrix from the pointto-point basis to the channel basis is described in details in the supplemental material [29]. Fig. 2(a) displays an example of matrix $\mathbf{S}$ in the channel basis recorded at frequency $f=0.36$ MHz. Despite its overall random appearance, one can see the residual ballistic wave-front that slightly emerges along the diagonal of the transmission matrices. The matrix $\mathbf{S}$ also exhibits long-range correlations that will account for the bimodal behavior of the transmission/reflection matrices.

Theoretically, energy conservation would imply that $\mathbf{S}$ is unitary. In other words, its eigenvalues should be distributed along the unit circle in the complex plane. The eigenvalues $s_{i}$ of the S-matrix at $f=0.36 \mathrm{MHz}$ are displayed in the complex plane in Fig. 2(b). The dispersion of these eigenvalues around the unit circle questions the validity of the energy conservation assumption. Yet duralumin is known for its weak absorption properties $\left(\sim 1.7 \mathrm{~dB} \cdot \mathrm{m}^{-1}\right.$ in the $0.1-0.5 \mathrm{MHz}$ frequency bandwidth [32]). Radiation losses through conversion of elastic waves into acoustic waves in surrounding air can also be neglected ( $\sim 1 \mathrm{~dB} \cdot \mathrm{m}^{-1}$ at $0.36 \mathrm{MHz}$ [33]). Actually, the non-unitarity of $\mathbf{S}$ is due to the experimental noise. Its magnitude, its nature and its various origins are described in details in the Supplemental material [29]. In the following, we compensate for this undesirable effect by building a virtual scattering matrix $\hat{\mathbf{S}}$ with the same eigenspaces as $\mathbf{S}$ but with normalized eigenvalues (see Fig. 2(b)), such that

$$
\hat{s}_{i}=s_{i} /\left|s_{i}\right|, \text { for } i=1, \cdots, N
$$

The phase information is thus unchanged and only the amplitude is normalized to meet the energy conservation requirement. We will show that this normalization based on energy conservation is essential to observe the open channels.

We first focus on the transmission eigenvalues $T$ and $\hat{T}$ computed from $\mathbf{S}$ and $\hat{\mathbf{S}}$, respectively. Their sum directly provides an estimation of the dimensionless conductance of the disordered slab: $g=\sum_{i} T_{i} \sim 8$ and $\hat{g}=\sum_{i} \hat{T}_{i} \sim 8.1$. 


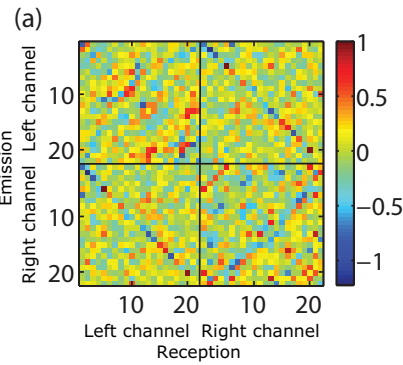

(b)

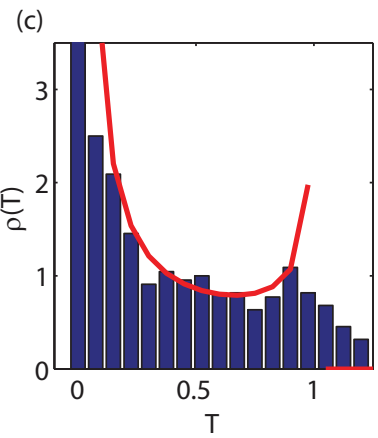

(d)

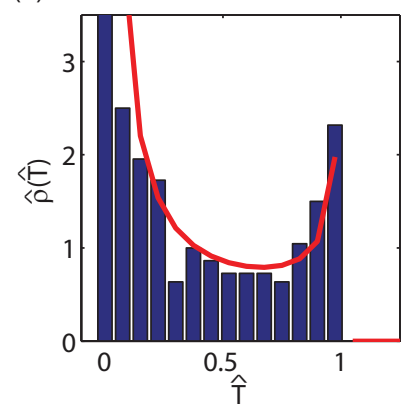

FIG. 2: a, Real part of the S-matrix measured at $f=0.36 \mathrm{MHz}$. The black lines delimit transmission and reflection matrices as depicted in Eq. 1. b, Eigenvalues $s_{i}$ (red dots) and $\hat{s}_{i}$ (blue squares) of the measured and normalized scattering matrices, $\mathbf{S}$ and $\hat{\mathbf{S}}$, respectively. The eigenvalues are displayed in the complex plane. The black continuous line denotes the unit circle. c-d, Transmission eigenvalue histograms, $\rho(T)$ and $\hat{\rho}(\hat{T})$, averaged over the frequency bandwidth. Both distributions are compared to the bimodal law $\rho_{b}$ (red continuous line, Eq. 3).

As we have access to $N=2 W / \lambda \sim 22$ independent channels, this yields a ratio $L / l^{*} \sim 2.75$ according to the Ohm's law. The distributions of the transmission eigenvalues, $\rho(T)$ and $\hat{\rho}(\hat{T})$, are estimated by averaging their histograms over the frequency bandwidth. Figs. 2(c)-2(d) show the comparison between these distributions and the bimodal law $\rho_{b}$ which is theoretically expected in the diffusive regime [7],

$$
\rho_{b}(T)=\frac{g}{2 T \sqrt{1-T}}
$$

Strictly speaking, our system is not in a fully diffusive regime ( $L \sim 2.75 l^{*}$ ). Yet, the measured eigenvalue distribution $\rho(T)$ is in correct agreement with the bimodal law $\rho_{b}(T)$ with a large peak around $T=0$ associated to the closed channels and a smaller peak around $T=1$ associated to the open channels (Fig. 2(c)). Nevertheless, some channels exhibit a transmission coefficient superior to 1 , which violates energy conservation. This is explained by the non-unitarity of $\mathbf{S}$ pointed out in Fig. 2(b). On the contrary, after normalization of $\mathbf{S}$ (Eq. 2), all transmission eigenvalues $\hat{T}$ are repelled below 1 and the eigenvalue distribution $\hat{\rho}(\hat{T})$ closely follows the expected bimodal law (Fig. 2(d)). This confirms that the unitarity of $\mathbf{S}$ is decisive and that experimental noise can prevent from recovering the bimodal law experimentally. In the supplemental material [29], a numerical simulation confirms that the nor- malization of $\mathbf{S}$ (Eq. 2) allows to retrieve almost completely the open channels, thus cancelling the detrimental effect of noise. We will now prove it experimentally by probing the open eigenchannels deduced from $\mathbf{S}$ and $\hat{\mathbf{S}}$.

Whereas the eigenvalues of $\mathbf{t t}^{\dagger}$ yield the transmission coefficients of each eigenchannel, the corresponding eigenvector provide the combination of incident modes that allow to excite this specific channel. Hence, the wave-field associated to each eigenchannel can be measured by backpropagating the corresponding eigenvector. To that aim, the scattering medium is scanned with the interferometric optical probe [29]. As a reference, the wave-field induced by an incident plane-wave is shown in Fig. 3(a). Fig. 3(e) plots the corresponding intensity averaged along the plate section ( $y$-axis) as a function of depth $x$. Fig. 3(b) displays the wave-field associated to a closed eigenchannel $(T \sim 0)$. The wave is fully reflected back to the left. This shows that the incoming wavefield has been successfully tailored to fit the randomness of the waveguide, making it a nearly perfectly reflecting medium. Beyond one transport mean-free path, the intensity decreases very rapidly in agreement with numerical simulations [15]. Figs. 3(c)-3(d) display the propagation of open eigenchannels ( $T \sim 1$ and $\hat{T} \sim 1$ ) deduced from the measured and normalized matrices $\mathbf{S}$ and $\hat{\mathbf{S}}$, respectively. Both wave-fields clearly show the contructive interferences that help the wave to find its way through the maze of disorder. The corresponding intensity profiles are shown in Figs. 3(g)-3(h). In both cases, the field intensity increases inside the medium. For the eigenchannel derived from the S-matrix, the intensity measured at the output of the scattering medium is smaller than at the input (Fig. 3(g)). Experimental noise in the $\mathbf{S}$-matrix prevents from addressing a fully opened channel. On the contrary, Fig. 3(h) illustrates how nicely the normalized $\hat{\mathbf{S}}$-matrix gives access to a fully open eigenchannel with equal intensities at the input and output of the scattering medium. The incoming wavefield has been successfully designed to make the scattering slab completely transparent.

From a more general point of view, we want to highlight that being able to address the open eigenchannels is the key to make the best of a scattering medium, for many applications. For instance in digital telecommunication, the maximum information that can be conveyed with no error is Shannon's capacity $C$. $C$ is actually determined by the number $g$ of open eigenchannels, in a multiple input-multiple output scheme [24, 34]. Hence a complete channel control allows to reach the maximum information transfer rate. An other example is wave focusing through a multiple scattering medium, whether it be by time-reversal [35, 36], phase conjugation [37] or wavefront-shaping optimization as the one used by Vellekoop and Mosk [38]. At one frequency, the maximum contrast between the focal spot and the background intensity at the output is given by the effective number $N_{\text {eff }}=3 g / 2$ of channels contributing to the transmitted field $[13,39]$. Again, the maximum is reached when the channel control is complete and the bimodal law is retrieved. Hence, the phase conjugation process is fully optimized only 
(a)

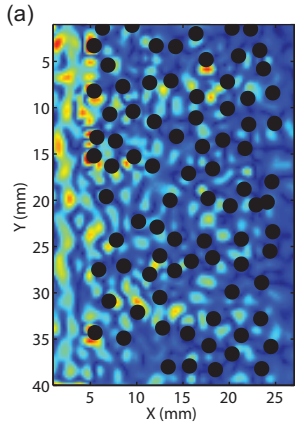

(e)

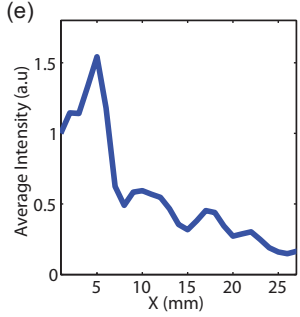

(b)
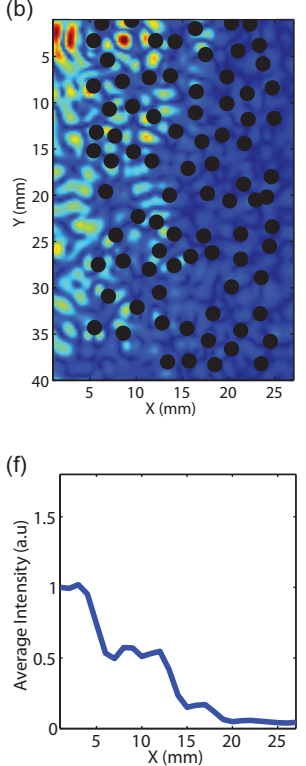

(c)
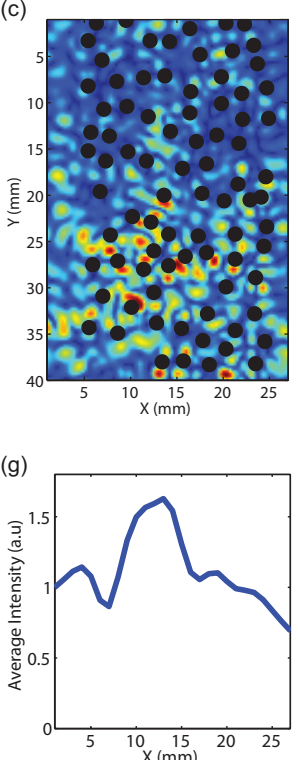

(d)
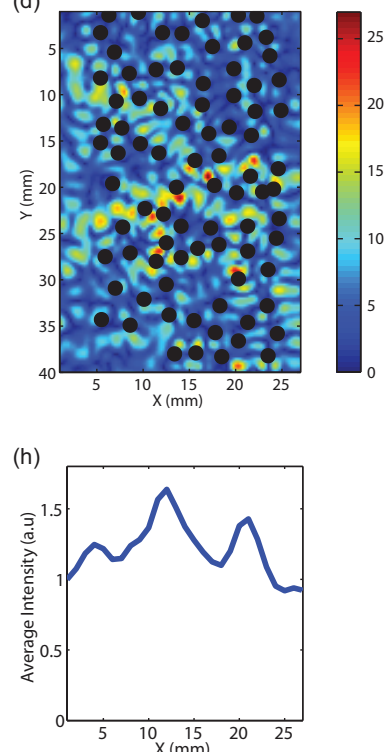

FIG. 3: Absolute value of the wave-field in the scattering medium at $f=0.36 \mathrm{MHz}$ associated to a, an incident plane wave, $\mathbf{b}$, a closed eigenchannel, c, an open eigenchannel deduced from the measured S-matrix and d, an open eigenchannel deduced from the normalized $\hat{\mathbf{S}}$ matrix. The corresponding intensities averaged over the wave guide section (y-axis) are shown versus depth $x$ in lower panels eh. They are all normalized by the intensity at the plane of sources $(x=0)$. if all the open eigenchannels are properly addressed.

In summary, this experimental study allows the direct observation of fully open and closed eigenchannels through a scattering medium. In agreement with theoretical predictions, transmission eigenvalues across a disordered system are shown to follow a bimodal law. The wave-field associated to each eigenchannel is measured and illustrates the remarkable interference mechanisms induced by disorder. From a fundamental point of view, such measurements will allow to check a whole set of RMT predictions that could not have been confronted to experiment so far. The transition towards Anderson localization should lead to an extinction of fully opened eigenchannels $[4,5,7]$ and the occurence of necklace states [3]. From a more practical point of view, this work paves the way towards an optimized control of wave-fronts through scattering media in all fields of wave physics, whether it be for communication, imaging, focusing, absorbing, or lasing purposes.

The authors wish to thank M. Davy, G. Lerosey, S. Rotter, S. Popoff, and A. Goetschy for fruitful discussions, as well as A. Souilah for his technical help. The authors are grateful for funding provided by LABEX WIFI (Laboratory of Excellence within the French Program Investments for the Future, ANR-10-LABX-24 and ANR-10-IDEX-0001-02 PSL*). B.G. acknowledges financial support from the French "Direction Générale de l'Armement"(DGA).

* Electronic address: alexandre.aubry@espci.fr [1] P. W. Anderson, Phys. Rev. 109, 1492 (1958).
[2] A. Lagendijk, B. A. van Tiggelen, and D. S. Wiersma, Physics Today 62, 24 (2009).

[3] J. B. Pendry, Physics 1, 20 (2008).

[4] O. N. Dorokhov, Sol. St. Comm. 51, 381 (1984).

[5] Y. Imry, Europhys. Lett. 1, 249 (1986).

[6] J. B. Pendry, A. MacKinnon, and A. B. Pretre, Physica A 168, 400 (1990).

[7] C. W. J. Beenakker, Rev. Mod. Phys. 69, 731 (1997).

[8] A. P. Mosk, A. Lagendijk, G. Lerosey, and M. Fink, Nature Photon. 6, 283 (2012).

[9] R. Sprik, A. Tourin, J. de Rosny, and M. Fink, Phys. Rev. B 78, 012202 (2008).

[10] A. Aubry and A. Derode, Phys. Rev. Lett. 102, 084301 (2009).

[11] A. Aubry, L. A. Cobus, S. E. Skipetrov, B. A. van Tiggelen, A. Derode, and J. H. Page, Phys. Rev. Lett. 112, 043903 (2014).

[12] Z. Shi and A. Z. Genack, Phys. Rev. Lett. 108, 043901 (2012).

[13] M. Davy, Z. Shi, J. Wang, and A. Z. Genack, Opt. Express 21, 10367 (2013).

[14] S. M. Popoff, G. Lerosey, R. Carminati, M. Fink, A. C. Boccara, and S. Gigan, Phys. Rev. Lett. 104, 100601 (2010).

[15] M. Kim, Y. Choi, C. Yoon, W. Choi, J. Kim, Q.-H. Park, and W. Choi, Nature Photon. 6, 583 (2012).

[16] S. M. Popoff, A. Aubry, G. Lerosey, M. Fink, A. C. Boccara, and S. Gigan, Phys. Rev. Lett. 107, 263901 (2011).

[17] Y. Choi, T. R. Hillman, W. Choi, N. Lue, R. R. Dasari, P. T. C. So, W. Choi, and Z. Yaqoob, Phys. Rev. Lett. 111, 243901 (2013).

[18] S. Feng and P. A. Lee, Science 251, 633 (1991).

[19] W. Choi, A. P. Mosk, Q. H. Park, and W. Choi, Phys. Rev. B 83, 134207 (2011).

[20] S. Rotter, P. Ambichl, and F. Libisch, Phys. Rev. Lett. 106, 120602 (2011).

[21] Y. D. Chong and A. D. Stone, Phys. Rev. Lett. 107, 163901 (2011).

[22] C. W. J. Beenakker and M. Büttiker, Phys. Rev. B 46, 1889 (1992).

[23] M. Henny, S. Oberholzer, C. Strunk, and C. Schönenberger, Phys. Rev. B 59, 2871 (1999).

[24] A. Goetschy and A. D. Stone, Phys. Rev. Lett. 111, 063901 
(2013).

[25] S. M. Popoff, A. Goetschy, S. F. Liew, A. D. Stone, and H. Cao, Phys. Rev. Lett. 112, 133903 (2014).

[26] S. Krishnaswami, Ultrasonic Nondestructive Evaluation (CRC Press, Boca Raton, FL, 2004), chap. Theory and applications of laser-ultrasonic techniques, pp. 435-494.

[27] D. Royer and E. Dieulesaint, Elastic waves in solids I (Springer-Verlag, Berlin, 2000).

[28] O. Diligent, T. Grahn, A. Boström, P. Cawley, and M.J. Lowe, J. Acoust. Soc. Am. 112, 2589 (2002).

[29] See Supplemental Material for a detailed description of the channel basis construction, the origins of the experimental noise, a numerical proof for the validity of the S-matrix normalization and details about the measurement of wave-fields associated to each eigenchannel.

[30] M. C. Cross and R. Lifshitz, Phys. Rev. B 64, 085324 (2001).

[31] D. H. Santamore and M. C. Cross, Phys. Rev. B 66, 144302 (2002).
[32] C-H. Hsieh and Y-F. Chang, Terr. Atmos. Ocean Sci. 7, 31 (1996).

[33] Radiation losses have been estimated numerically with the DISPERSE software [40]

[34] A. Derode, A. Tourin, J. de Rosny, M. Tanter, S. Yon, and M. Fink, Phys. Rev. Lett. 90, 014301 (2003).

[35] A. Derode, P. Roux, and M. Fink, Phys. Rev. Lett. 75, 4206 (1995).

[36] M. Fink, Physics Today 50, 34 (1997).

[37] Z. Yaqoob, D. Psaltis, M. S. Feld, and C. Yang, Nature Photon. 2, 110 (2008).

[38] I. M. Vellekoop and A. P. Mosk, Opt. Lett. 32, 2309 (2007).

[39] I. M. Vellekoop and A. P. Mosk, Phys. Rev. Lett. 101, 120601 (2008).

[40] B. Pavlakovic and M.J. Lowe, Disperse Software, v.2.0.16 (Mechanical Engineering, Imperial College, London, 2005). 